\title{
A Treatment Protocol for Restoring Occlusal Vertical Dimension Using an Overlay Removable Partial Denture as an Alternative to Extensive Fixed Restorations: A Clinical Report
}

\author{
Mit B. Patel ${ }^{1}$ and Sompop Bencharit ${ }^{2, *}$
}

\author{
${ }^{I}$ Dental Resident, Advanced General Dentistry Program, Fayetteville Veterans Affairs Medical Center; and Former \\ Senior DDS Student, School of Dentistry, University of North Carolina at Chapel Hill, Chapel Hill, NC 27599, USA \\ ${ }^{2}$ Department of Prosthodontics, School of Dentistry, and Department of Pharmacology, School of Medicine, University \\ of North Carolina at Chapel Hill, Chapel Hill, NC 27599, USA
}

\begin{abstract}
Treatment options for patients with severe attrition resulting in reduced occlusal vertical dimension are often limited to fixed prosthesis to reestablish proper occlusal vertical dimension and functional occlusion. In some cases such as when there are limited finances, minimal esthetic concerns, and medical considerations fixed prosthesis may not be the ideal treatment option. Overlay removable partial dentures (ORPDs) can be used as a provisional or interim prosthesis as well as permanent prosthesis in these cases. While ORPDs can provide a reversible and relatively inexpensive treatment for patients with a significantly compromised dental status, there is not much scientific evidence in the literature on ORPDs. Most studies published on ORPDs to date are primarily reviews and clinical reports. In this article, literatures on ORPDs are summarized and a patient treated with interim and permanent ORPDs is presented. This article reviews previously published literatures on the use of ORPDs. Indications, advantages and disadvantages are discussed. Treatment protocol with an example of the prosthodontic treatment of a patient with severely worn dentition with an interim ORPD and later a permanent ORPD are discussed in details.
\end{abstract}

Keywords: Overlay removable partial denture, worn dentition, vertical dimension, oral rehabilitation.

\section{INTRODUCTION}

Overlay removable partial dentures (ORPDs), a subset of overdentures, are often referred to as an RPD that has part of their components covering the occlusal surface of the abutment teeth to restore them into a functional occlusion [1,2]. Unlike conventional overdentures that only a few millimeters of coronal tooth structure of the abutment teeth are left supragingivally, there is usually at least one-third or half of tooth structure remaining in ORPD situation. This remaining tooth structure is often visually exposed contributing to esthetic challenge compared to the conventional overdenture where the abutment teeth are completely covered [3]. ORPDs are most often used as interim prostheses prior to fixed full mouth restorations or prior to a treatment combination of permanent fixed and removable prostheses $[4,5]$. In addition to interim use of ORPDS, they can also be used as permanent prostheses [6,7]. While ORPDs are used widely, there is not much scientific evidence on ORPDs in the literature. Unlike tooth-retained conventional overdentures or implant-retained overdentures, there are virtually no longitudinal studies or clinical trials of ORPDs. The only evidences in the literature on ORPDs are mostly in textbooks, narrative reviews, and clinical reports. This article therefore briefly

*Address correspondence to this author at the CB\#7450, Department of Prosthodontics, School of Dentistry, University of North Carolina at Chapel Hill, Chapel Hill, NC 27599-7450, USA; Tel: 919-843-8734; Fax: 919-9663821; E-mail: Sompop_Bencharit@dentistry.unc.edu reviews indications for ORPD in current literature and presents a clinical scenario on the use of ORPDs as an interim and a permanent prosthesis in a patient with severe worn dentition.

There are perhaps three main indications for ORPDs. First, as an interim prosthesis, an ORPD is most often prescribed for a patient with reduced occlusal vertical dimension (VDO) due to moderate to severe worn dentition [8]. This pattern of tooth loss generally occurs when the occlusal wear of teeth occurs at a faster pace than the compensatory continuous eruption of teeth. However, there are some patients that compensatory tooth eruption can overcome lost of VDO. Increasing VDO with prostheses in this last group of patients can be difficult [8]. Careful evaluation of decreased VDO, by using evidences of loss of posterior support, history of wear, phonetics, interocclusal distance, and facial appearance, is probably one of the most important step in full mouth rehabilitation for these patients [8-10]. Patients with reduced VDO can use ORPDs instead of occlusal splint to accurately evaluate their proper VDO [1-7,10,11]. Unlike occlusal splint, ORPDs offer an advantage that patients can use them all the time even during normal function including speaking or eating [1-7,10,11]. Unlike occlusal splint, ORPDs offer an advantage that patients can use them all the time even during normal function including speaking or eating $[1-7,10,11]$. An interim ORPD provides a reversible treatment that allows validation of VDO and functional occlusion during phase I dental treatment including caries control, periodontal, surgical, and endodontic treatment [10,11]. 


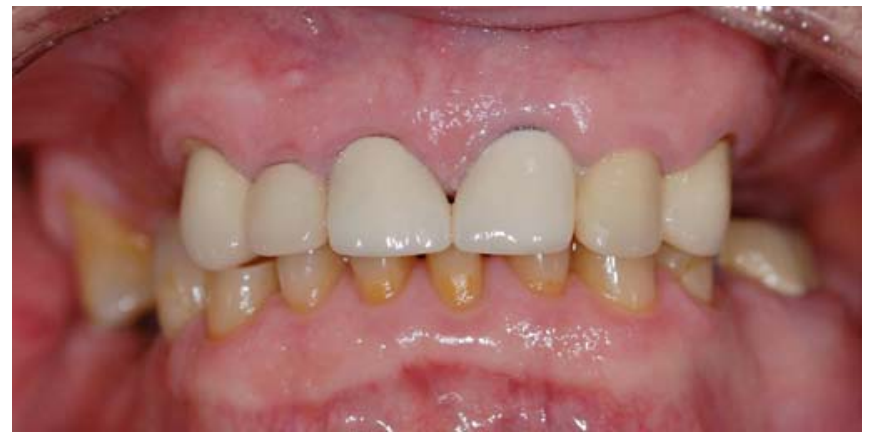

Fig. (1). Preoperative frontal view in maximal intercuspal position (MIP).

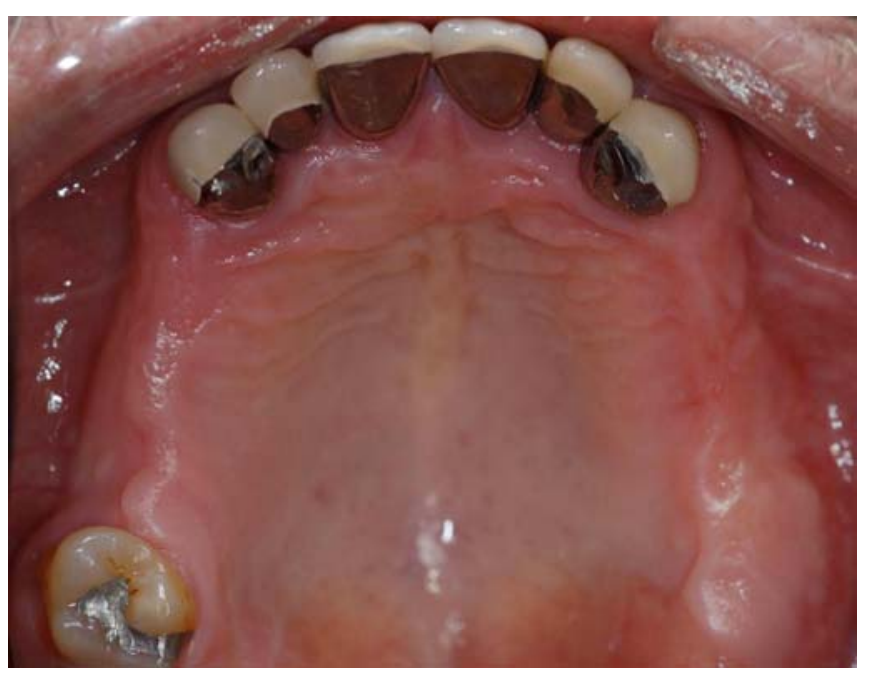

Fig. (2). Preoperative maxillary arch demonstrating Kennedy Class II Modification I with maxillary anterior porcelain fused to metal crowns.

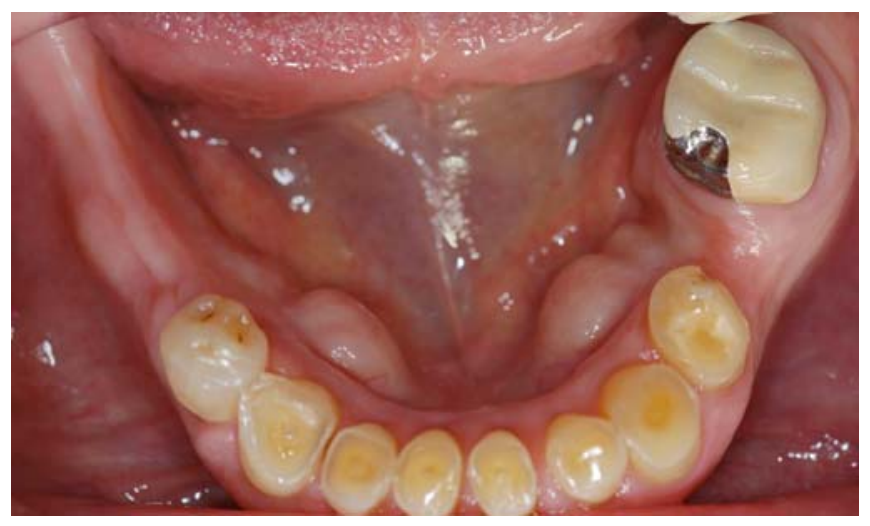

Fig. (3). Preoperative mandibular arch demonstrating Kennedy Class II Modification I with severe attrition of mandibular anterior teeth.

This validated VDO and functional occlusion by interim ORPDs can then be transferred to the final prostheses [1-7, 10-12].

Second, interim and permanent ORPDs are often prescribed to patients with severe dental and skeletal malocclusion [13-20]. These malocclusions can be resulting from cleft palate [13] class II or III skeletal malocclusion [14-16] or openbite or open occlusal relationship [1,17-20]. Several case reports show that ORPDs can help restore a functional occlusion with little or no surgical intervention. Del Castillo et al. suggest successful therapy with maxillary and mandibular ORPDs in patients with congenital and acquired anomalies such as posterior open occlusal relationships [18]. Gitt used an ORPD in a patient with cleft palate when the premaxilla is severe underdeveloped and disrupted [13]. Murray used an ORPD to restore a functional occlusion of a patient with severe skeletal class III malocclusion [14]. Radlanski and Freesmeyer reported using an ORPD to correct an openbite resulting from skeletal malocclusion [17]. Atobe et al. reported using an ORPD to correct an openbite resulting from multiple ankylosed teeth [19].

Third, the last common indication for ORPDs is medical or financial limitation for fixed prosthodontics [3-7,10, 21]. Some patients can have malocclusion or worn dentition that can ideally be restored with fixed prostheses perhaps in conjunction with orthodontic, periodontal, and surgical treatments. However, financial concerns or general medical problems could prevent these treatments $[3-7,10,21]$. The major advantages of ORPDs are that they are relatively simpler and less expensive than the fixed prosthetic option.

The following case report describes an example of the most common indication for ORPD, the rehabilitation of a patient with severe worn dentition with interim ORPDs and later permanent ORPDs. The patient has been treated with interim maxillary acrylic partial denture and mandibular overlay acrylic partial denture and later on maxillary RPD and mandibular cast overlay partial denture to correct reduced VDO. The therapy has improved function, facial esthetics, and muscle tone with reversible, non-invasive, and relatively inexpensive treatment. In several parameters, results are similar to extensive fixed restorations with compromises in esthetics and risks of material fracture [7, 22]. As patients continue to retain their dentition for more years, loss of tooth structure will be more prevalent, making ORPDs a viable alternative to fixed prosthodontic option.

\section{CLINICAL REPORT}

A 75 year old Caucasian male presents to UNC School of Dentistry Pre-doctoral Clinic for general dental care. The patient's medical history has no contraindications to dental treatment. The patient presented in partially edentulous state; Prosthodontic Diagnostic Index (PDI) classification IV; maxillary Kennedy Class II, modification I and mandibular Class II, modification I, with severe loss of occlusal vertical dimension (VDO) (Figs. 1, 2 and 3). The maxillary arch had been previously restored with fixed restorations at his existing VDO. There was severe attrition of mandibular anterior teeth. The patient had maxillary and mandibular removable partial dentures that had been in use for over 40 years. Patient's chief concern was to be able to regain proper function at lowest cost and without particular esthetic concern.

\section{Diagnosis and Treatment Planning}

Diagnostic casts were made and mounted on a semiadjustable articulator with facebow record and centric relation record. Patient informed that conventional RPDs could 


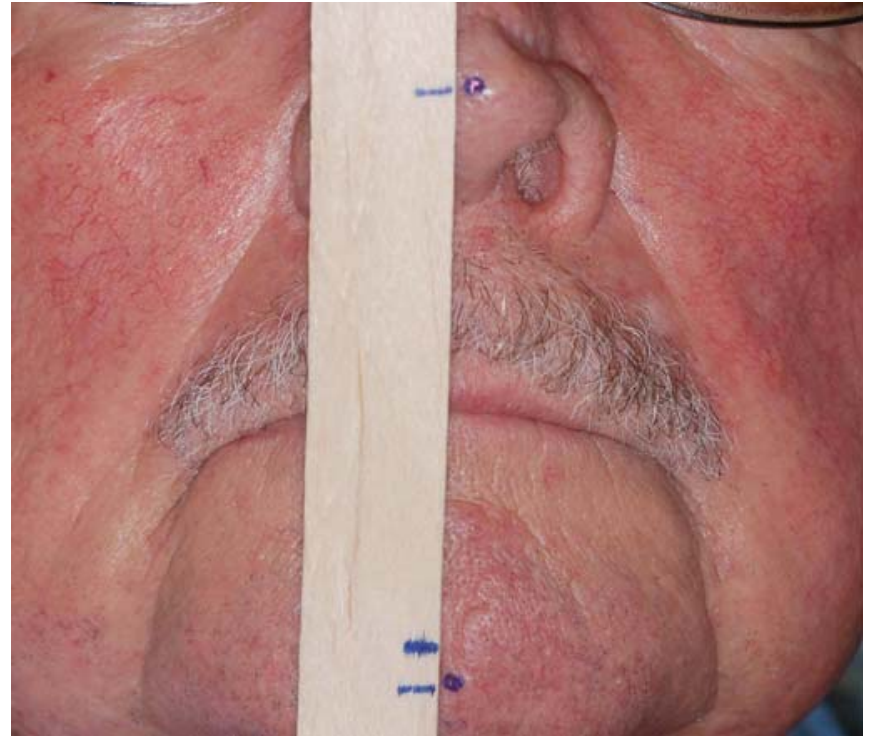

Fig. (4). Determination of occlusal vertical dimension (VDO).

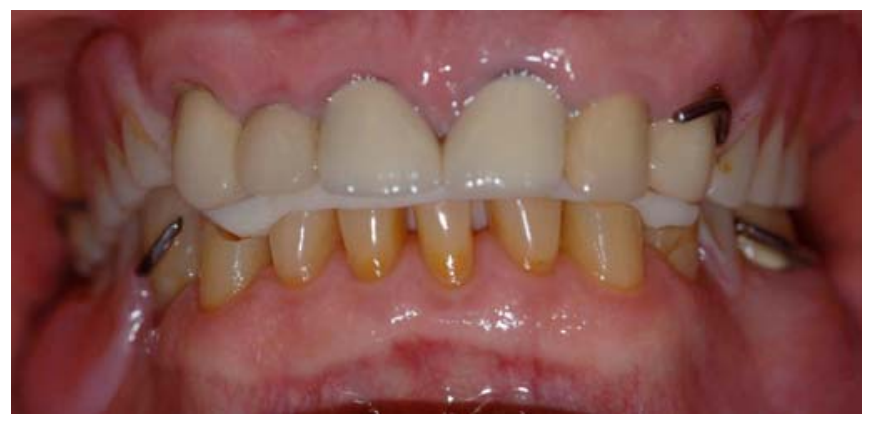

Fig. (5). Frontal view of interim prostheses in MIP.

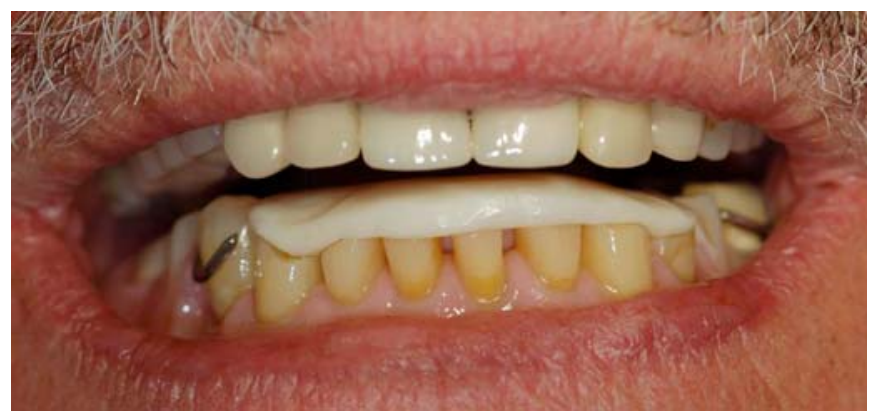

Fig. (6). Frontal view of interim prostheses with slight opening.

longer be fabricated due to severe attrition and decrease in VDO. Patient desired to maintain all remaining teeth. Patient had bilateral mandibular tori and was unwilling to undergo surgical removal. Treatment options presented to patient in the following order: [1] No treatment, [2] Crowns for mandibular anterior teeth to appropriate VDO followed by removable partial dentures, [3] Composite buildup of mandibular anterior teeth to appropriate VDO followed by RPDs, [4] Mandibular ORPD to appropriate VDO and maxillary RPD to ideal occlusion.

The patient did not have financial capacity for options $\# 1,2$, or 3 . The patient however was interested in option \#4.

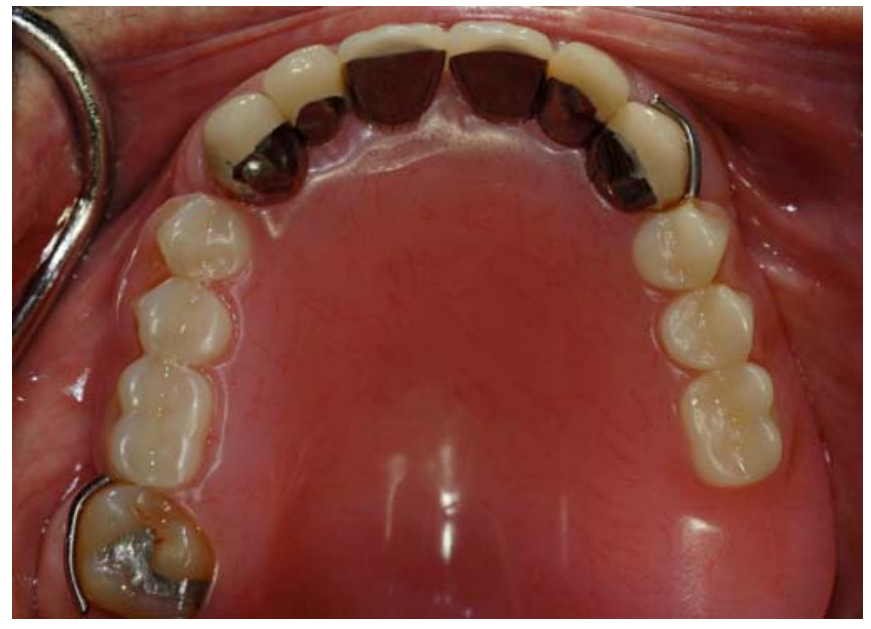

Fig. (7). Maxillary interim prosthesisbb.

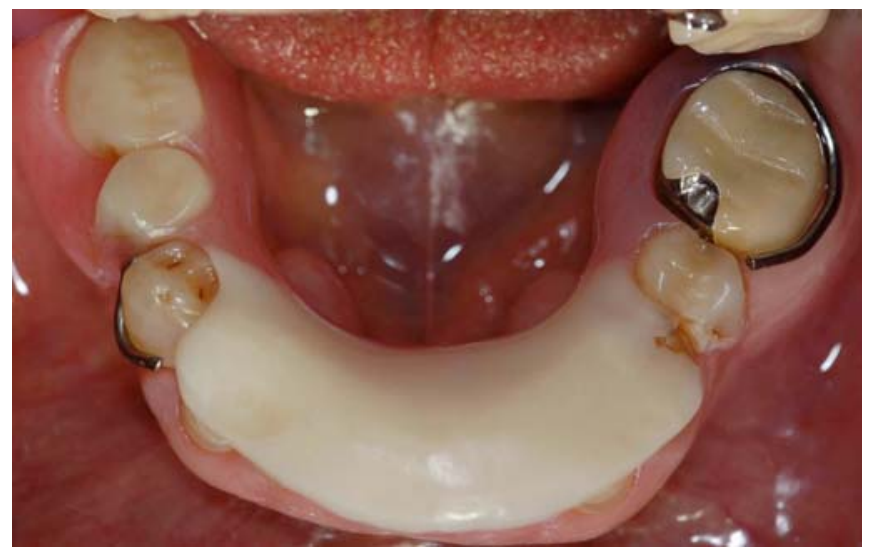

Fig. (8). Mandibular interim overlay partial denture.

The patient was informed that this was not the ideal treatment option and esthetics might be significantly compromised with this option. He was also informed that fabrication of his prostheses would require restoring his VDO with interim occlusal overlay prosthesis, and these interim prostheses would be required for at least 6 weeks prior to initiation of definitive cast partial dentures to determine if patient comfortable with the increased VDO. Patient was fully informed of all risks/benefits/alternatives and decided on therapy option \#4.

\section{Treatment Procedure}

The maxillary interim acrylic partial denture and mandibular overlay partial denture were fabricated after minimally preparing the occlusal surfaces of mandibular anterior teeth minimally to allow flat, smooth surface for mandibular RPD framework adaptation. Impressions were made with alginate (Jeltrate, Dentsply International, York, PA, USA) to fabricate definitive casts for the interim dentures. The appropriate VDO was determined using interocclusal distance, vertical dimension of rest (VDR) and sibilant sound (Figs. 48). These casts were crossed mounted with the diagnostic casts using occlusion rims and polyvinyl siloxane registration material (Regisil, Dentsply International, York, PA, USA) at the centric relation (CR) position. Wax setup of denture teeth were tried in to evaluate VDO using the VDR, 


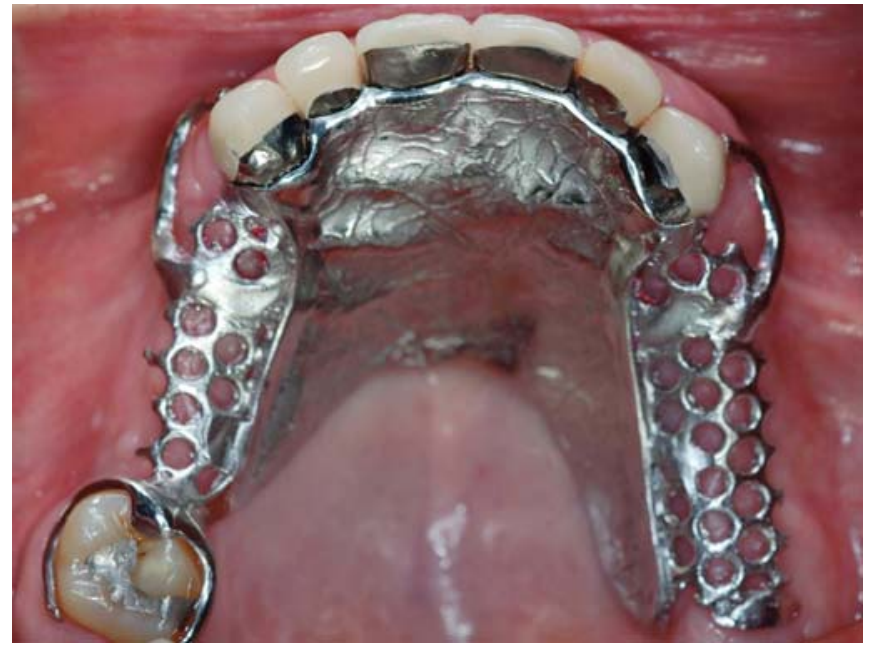

Fig. (9). Maxillary RPD framework.

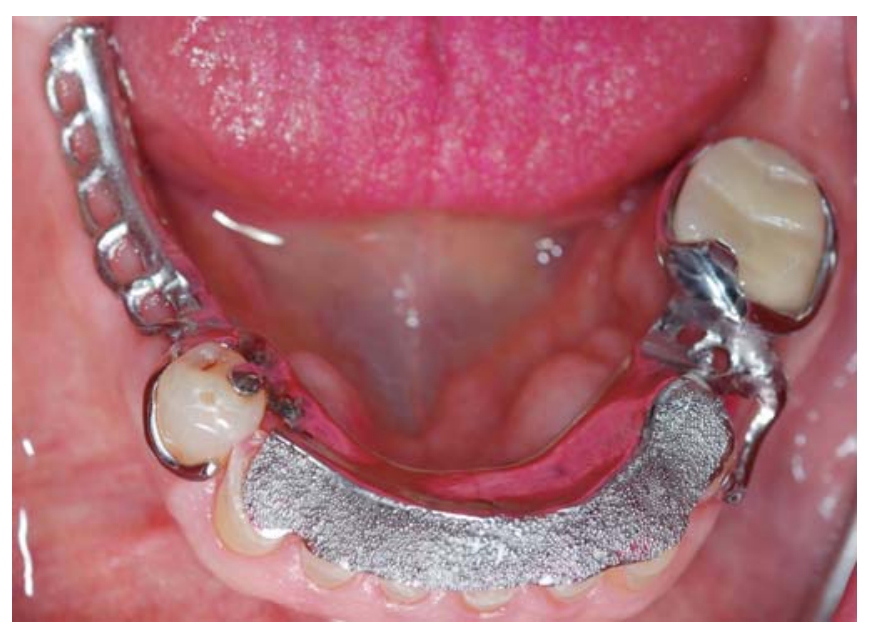

Fig. (10). Mandibular RPD framework.

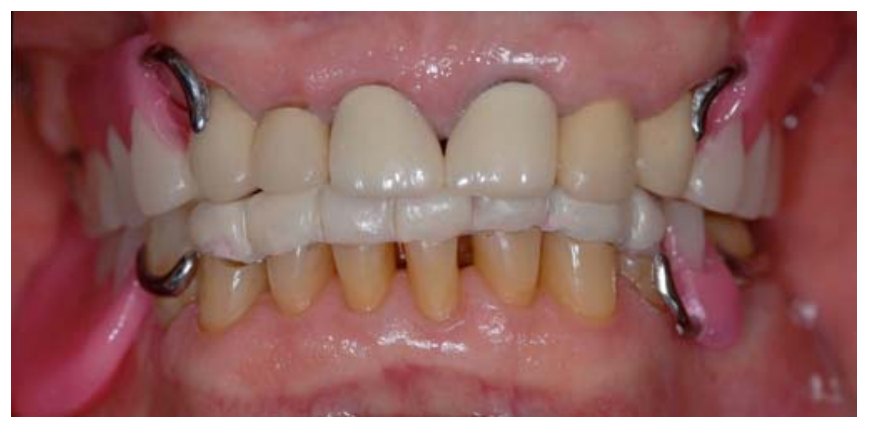

Fig. (11). Wax try-in.

interocclusal space, and phonetic methods. The interim dentures were then processed in heat-cured acrylic resin. Note that tooth color acrylic resin was used in the anterior portion of the mandibular interim denture. Laboratory remount and equilibration was performed prior to delivery. During the delivery visit, VDO and centric occlusion (CO) were verified. The dentures were adjusted to proper VDO and CO. The patient was instructed to wear these dentures all day and to remove them at night time. The patient was follow-up at

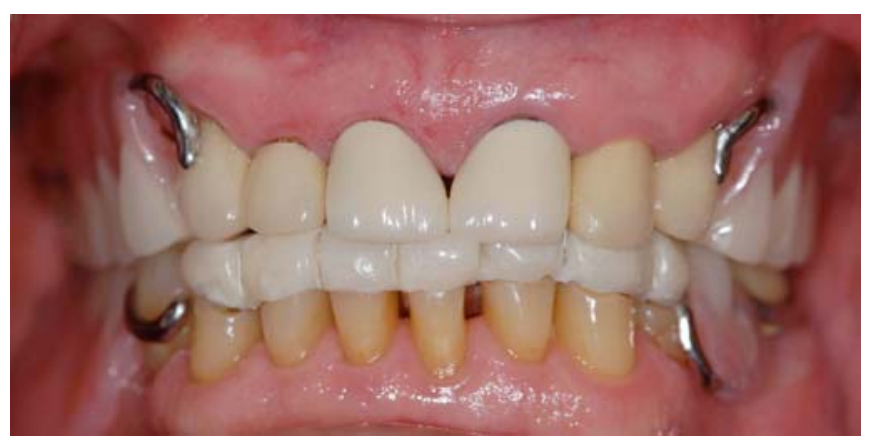

Fig. (12). Final prostheses in MIP.

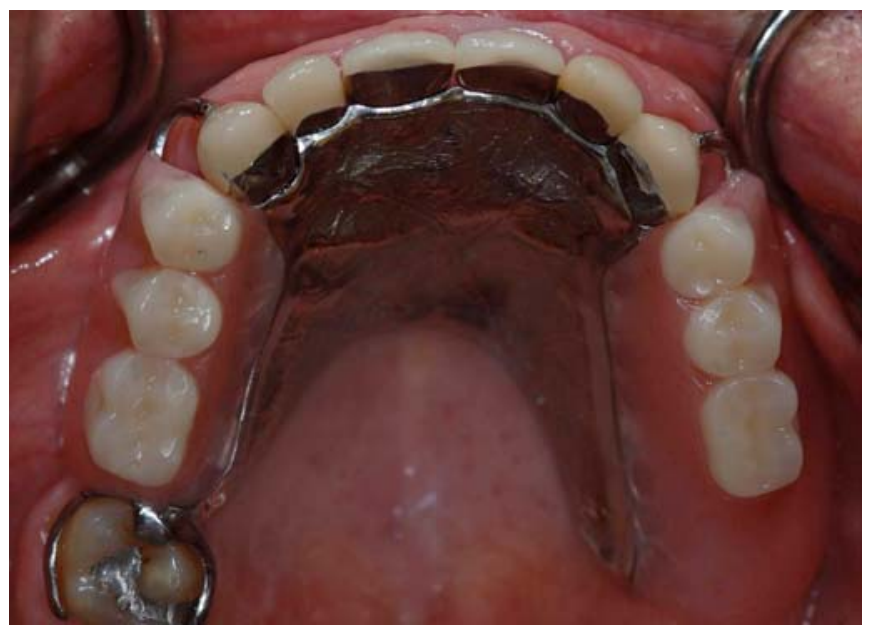

Fig. (13). Maxillary final prosthesis.

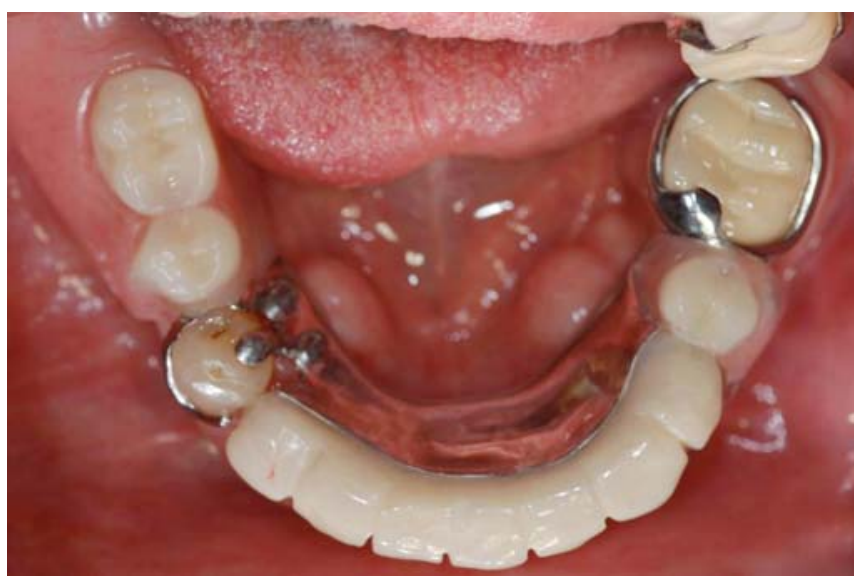

Fig. (14). Mandibular final prosthesis.

one-, four-, and six-week postoperative visits. At each visit minor adjustment of the denture base was made. However, the patient reported no muscle or TMJ tenderness. He also reported that he was comfortable and functioned well with these prostheses.

After eight weeks of using interim dentures, the VDO was again determined using the same techniques described earlier. It was then determined that another $2-\mathrm{mm}$ in the incisal region can be increased in the final prostheses. This dimension would also allow the space for metal framework and the acrylic teeth. (Figs. 9,10). Acrylic (Triad) anterior positioning jig with posterior polyvinyl siloxane were used 
in CR record. The final VDO was carefully determined in the wax try-in visit (Fig. 11). The final prostheses were delivered (Fig. 12-14). The patient was followed up at twentyfour hours, one week, one month, and finally six months. At each visit, the patient reported that he was comfortable with the prostheses and functioned well with them. He reported no symptom of muscle or TMJ tenderness.

\section{DISCUSSION}

Severely worn dentition is common problem seen in the population and restorative challenge for dentists. This condition is often associated with a reduction in occlusal vertical dimension (VDO). This case illustrates the plausibility of overlay removable partial denture (ORPD) rehabilitation of these patients when missing teeth and worn dentition are involved similar to previous reports $[2,6,7,11]$. Six months after delivery of the maxillary and mandibular partial, the patient reported no problems with the prosthesis. The patient was pleased with the retention, function, and even esthetics of the RPDs. Treatment time was lengthened only during the initial trial phase of the increase VDO. This trial phase was believed to allow neuromuscular adjustment to a change in VDO while also providing the patient to report satisfaction with function and esthetics. A similar removable occlusal overlay prosthesis was recommended for 6 to 8 weeks adaptation period for final fixed prosthesis in similar situations as well [8]. In our case, after six weeks of interim prostheses, we added two millimeters of VDO at the mandibular anterior area to the final prostheses after re-assessment of his VDO following 6 weeks of interim period. We determined at the framework try-in visit that his VDO could be increased and the patient reported no muscle or TMJ pain. He reported feeling comfortable at every postoperative visit. The procedure was relatively non-invasive which may be relevant during treatment planning since most patients with cases of severe attrition are seen in the elderly. Treatment costs were significantly less than all other options to restore severely worn dentition. Esthetic and functional limitations as well as potential risks for material fracture or debonding should be discussed with the patient when comparing to fixed restorations. While there are limited studies on the longevity of ORPDs, the risks of wear, fracture, or debonding of occlusal materials are likely to be a primary failure. This failure may be prevented by using composite resin or metal materials. Garcia and Bohnenkamp described the use of composite resin for RPD that can be applicable to ORPD [23]. Similarly, Imbery et al described the fabrication of gold occlusal surface [24]. An in vitro study suggests that composite resin is perhaps a good option for occlusal surface of ORPD where the underlying framework is rigid, while acrylic resin should be used in the denture base area where the framework is less rigid and subjected to flexural forces [25]. In this case, discuss about possible wear, fracture, and debonding of materials were discussed in details with the patient prior to any treatment. Acrylic resin and composite resin were selected for the interim and the permanent ORPDs, respectively; with the reason that both of these materials are relatively inexpensive and easy to fix if wear or fracture occurs. Regular recall and maintenance visits are therefore essential to ensure the long term success of ORPDs. In addition, the patient should be instructed and reinforced oral hygiene and denture hygiene with occlusal overlay prosthesis.

\section{CONCLUSIONS}

This case describes the use of overlay removable partial dentures to restore occlusal vertical dimension (VDO) in patients with severely worn dentition, specifically attrition of mandibular anterior teeth. In patients with limited finances, low esthetic concerns, or medical limitations, this may be a viable or often the only treatment option. Irrespective of the aforementioned factors, all patients with severely worn dentition with decreased VDO and missing teeth should be presented with this treatment option.

\section{ACKNOWLEDGEMENT}

The University of North Carolina at Chapel Hill's Office of the Vice Chancellor for Research and Economic Development provided support for open access publication.

\section{REFERENCES}

[1] Farmer JB, Connelly ME. Treatment of open occlusions with onlay and overlay removable partial dentures. J Prosthet Dent 1984; 51: 300-3.

[2] Gankerseer EJ. Case report: a new technique for the treatment of the severely worn dentition. Restorative Dent 1987; 3: 13-14.

[3] Windchy AM, Morris JC. An alternative treatment with the overlay removable partial denture: a clinical report. J Prosthet Dent 1998; 79: 249-53.

[4] Watson RM. The role of removable prostheses and implants in the restoration of the worn dentition. Eur J Prosthodont Restor Dent 1997; 5: 181-6.

[5] Zarati S, Ahmadian L, Arbabi R. A transitional overlay partial denture for a young patient: a clinical report. J Prosthodont 2009; 18: 76-9.

[6] Lee H, Oster C. A technique to fabricate metal occlusal surfaces for the overlay removable partial denture. J Prosthet Dent 2006; 96: 456-7.

[7] Guttal S, Narendra PP. Cast titanium overlay denture for a geriatric patient with a reduced vertical dimension.Gerodontology 2005; 22: 242-5.

[8] Turner KA, Missirlian DM. Restoration of the extremely worn dentition. J Prosthet Dent 1984; 52: 467-74.

[9] Verrett RG. Analyzing the etiology of an extremely worn dentition. J Prosthodont 2001; 10: 224-33.

[10] Jahangiri L, Jang S. Onlay partial denture technique for assessment of adequate occlusal vertical dimension: a clinical report. J Prosthet Dent 2002; 87: 1-4.

[11] Chu FC, Siu AS, Newsome PR, Chow TW, Smales RJ. Restorative management of the worn dentition. 4 Generalized toothwear. Dent Update 2002; 29: 318-24.

[12] Almog DM, Ganddini MR. Maxillary and mandibular overlay removable partial dentures for restoration of worn teeth. A three year follow-up. NY State Dent J 2006; 72: 32-5.

[13] Gitt I. Prosthetic rehabilitation of surgically and orthodontically pretreated patients with clefts. Stomatol DDR 1980; 30: 1-7.

[14] Murray CG. The prosthodontic rehabilitation of selected adults class III malocclusions. J Oral Rehabil 1979; 6: 147-52.

[15] Taylor RM. Vitallium removable overlays for deep overbitecase report. NZ Dent J 1985; 81: 28-9.

[16] Sakar O, Beyli M, Marsan G. Combined prosthodontic and orthodontic treatment of a patient with a Class III skeletal malocclusion: a clinical report. J Prosthet Dent 2004; 92: 224-8.

[17] Radlanski RJ, Freesmeyer WB. Bilateral open bite in dicygotic twins. A combined orthodontic-prosthetic approach. J Orofac Orthop 2002; 63: 339-47.

[18] Del Castillo R, LaMar F, Ercoli C. Maxillary and mandibular overlay removable partial dentures for the treatment of posterior openocclusal relationship: A clinical report. J Prosthet Dent 2002; 87: 587-92. 
[19] Atobe M, Sekiya T, Tamura K, Hamada Y, Nakamura Y. Severe lateral open bite caused by multiple ankylosed teeth: a case report. Oral Surg Oral Med Oral Pathol Oral Radiol Endod 2009; 107: e14-20.

[20] Glyn Jones JC, Basker RM. Restorative treatment of a bilateral open bite. Dent Update 1983; 10: 511-4.

[21] Gornitsky M, Hammouda W, Rosen H. Rehabilitation of a hemophiliac with implants: a medical perspective and case report. J Oral Maxillofac Surg 2005; 63: 592-7.

[22] Kolodney H Jr, Akerly WB. A composite resin veneer occlusal surface on an overlay partial denture. Compendium 1991; 12: 6670.
[23] Garcia LT, Bohnenkamp DM. The use of composite resin in removable prosthodontics. Compend Contin Educ Dent 2003; 24: 688-90, 692-94.

[24] Imbery TA, Evans DB, Koeppen RG. A new method of attaching cast gold occlusal surfaces to acrylic resin denture teeth. Quintessence Int 1993; 24: 29-33.

[25] Kountouras CG, Howlett JA, Pearson GJ. Flexural and thermal cycling of resins for veneering removable overlay dentures. J Dent 1999; 27: 367-72.

(C) Patel and Bencharit; Licensee Bentham Open.

This is an open access article licensed under the terms of the Creative Commons Attribution Non-Commercial License (http: //creativecommons.org/licenses/by-nc/3.0/) which permits unrestricted, non-commercial use, distribution and reproduction in any medium, provided the work is properly cited. 